\title{
Prevalência dos sintomas de estresse e ansiedade em graduandos de enfermagem durante atividades acadêmicas remotas
}

\section{Prevalence of stress and anxiety symptoms in nursing graduates during remote academic activities}

\author{
Gabrielly Viana Pusch ${ }^{1 *}$, Franciele Batista do Nascimento ${ }^{1}$, Aliny de Lima Santos ${ }^{1}$, Ivi Ribeiro \\ Back $^{1}$, Ludmila Lopes Maciel Bolsoni ${ }^{1}$
}

\begin{abstract}
RESUMO
O objetivo dessa pesquisa foi estimar a prevalência de sintomas de estresse em graduandos de enfermagem, em decorrência da situação transitada para aulas remotas, devido a COVID-19 e isolamento social. O estudo descritivo, transversal com abordagem quantitativa, entrevistou 75 graduandos de enfermagem em uma instituição de ensino privada. Utilizou-se três questionários para levantamento de dados sociodemográfico e psicossociais, rendimento acadêmico e Perceived Stress Scale (PSS), sendo aplicado de forma online e analisado por estatística descritiva. Entre os entrevistados predominou o sexo feminino (92\%), com idade de 16 à 25 anos (72\%), sendo que a maioria não realiza atividade física (64\%) e possui 6 à 8 horas diárias de sono $(70,7 \%)$, quanto ao rendimento acadêmico, as notas mantiveram-se elevadas. Em relação a PSS, a mediana da somatória de pontos correspondeu a 34, considerando assim, nível médio de estresse (72\%). Observou-se desta forma, a presença de sintomas de estresse percebido, evidenciando maior necessidade de conscientização e incentivo a terapias psicológicas, contribuindo assim, para possíveis melhorias, a fim de minimizar agravos futuros, melhorando a disposição para aprender e qualidade de vida.
\end{abstract}

Palavras-chave: Estresse Psicológico; Estudantes de Enfermagem; Isolamento Social.

\begin{abstract}
The objective of this research was to estimate the prevalence of stress symptoms in nursing students, as a result of the situation transferred to remote classes, due to COVID-19 and social isolation. The descriptive, cross-sectional study with a quantitative approach interviewed 75 nursing students in a private educational institution. Three questionnaires were used to survey sociodemographic and psychosocial data, academic performance and the Perceived Stress Scale (PSS), which were applied online and analyzed using descriptive statistics. Among the respondents, females predominated (92\%), aged 16 to 25 years (72\%), most of whom do not perform physical activity (64\%) and have 6 to 8 hours of sleep a day (70.7\%), as for academic performance, the grades remained high. Regarding PSS, the median of the sum of points corresponded to 34 , thus considering the average level of stress (72\%). Thus, the presence of symptoms of perceived stress was observed, showing a greater need for awareness and encouragement of psychological therapies, thus contributing to possible improvements in order to minimize future harm, improving the willingness to learn and quality of life.
\end{abstract}

Keywords: Stress, Psychological; Students, Nursing; Social Isolation.

\footnotetext{
${ }^{1}$ Campus Maringá-PR, Universidade Unicesumar 1. *E-mail: gabriellypusch2@gmail.com
} 


\section{INTRODUÇÃO}

A pandemia atual provocada pela COVID-19, surgiu na cidade de Wuhan, na China, notificada inicialmente em 31 de dezembro de 2020 pela OMS (CASTELLA et al., 2020), devido a manifestação de um novo agente etiológico da família do coronavírus, conhecido como SARS-CoV-2. A transmissão ocorre por gotículas de saliva, podendo ser de forma direta e indireta, através da tosse, espirro, fala, além de objetos e superfícies contaminadas. Devido a rápida transmissão e grande número de novos casos e óbitos, provocou uma emergência em saúde (OPAS, 2020), sendo necessário um distanciamento entre as pessoas, o que ocasionou em isolamento social. Decorrente disso, o Ministério da Educação em relação aos estudantes decretou, através da portaria $\mathrm{N}^{\circ} 343,17$ de março de 2020, a mudança das aulas presenciais para digitais, subentendidas como atividades remotas, a fim de evitar aglomeração de pessoas e novas transmissões (BRASIL, MEC, 2020).

Segundo estudos, cerca de $73 \%$ das pessoas estão passando por algum grau de estresse, devido ao isolamento social, que tem provocado mudanças no estilo de vida das pessoas de modo geral, ocasionando limitações. Muitas destas pessoas estão saindo apenas para cumprirem com suas necessidades básicas, como comprar alimentos e medicamentos (BEZERRA et al., 2020). Dentre essas pessoas então os estudantes universitários, os quais antes da pandemia tinham normalmente aulas presenciais, e por consequência passaram a ter atividades remotas (LIMA, 2020), o que também tem acarretado em estresse (BRASIL, MEC, 2020; BEZERRA et al., 2020).

O estresse é considerado um enfrentamento psicológico, evidenciado por um momento em que a pessoa excede suas limitações rotineiras, ou seja, passa por alguma situação, não conseguindo se adaptar e saindo do seu equilíbrio homeostático (LIPP, 1997). Com aprofundamento dos estudos para avaliações de sintomas de estresse, o mesmo apresentava-se em três fases, que seriam alerta, resistência e exaustão. Essas ainda são utilizadas, porém identificou-se a existência de mais uma fase, a quase-exaustão (LIPP, 2001).

O estresse começa a se tornar positivo na fase de alerta, consistindo em uma liberação de adrenalina no organismo, a fim de realizar reação de luta e fuga, obtendo um sentimento de satisfação. Se o estresse persiste provocando desgaste emocional e cansaço, o indivíduo entra na fase de resistência, porém se não consegue adaptação, ocorre uma 
quebra na resistência física, devido esgotamento de reserva de energia, gerando doenças, caracterizando a fase de quase-exaustão. Já a fase final do estresse é mais agravante, pois afeta órgãos mais vulneráveis, surgindo doenças como infarto agudo do miocárdio, psoríase, aumento da pressão arterial, câncer, entre outras (LIPP, 2001).

Em diversos estudantes de graduação é notável que desequilíbrios na qualidade de vida promovem descontrole emocional e cansaço, consequentes de limitações, provocadas por demandas acadêmicas que sobrecarregam, gerando assim um estresse pelas mudanças (LOPES et al., 2019).

A sobrecarga acadêmica também evidenciada em discentes de enfermagem pode provocar mal aprendizado, culminando em um futuro profissional desqualificado, podendo prejudicar a assistência prestada ao paciente. Isso inclusive está relacionado a cobrança realizada pelo professor e a forma metodológica de aplicação das atividades, envolvendo a relação entre professor e aluno, a persistência do enfrentamento em querer ser um bom discente e concluir aquilo que é solicitado a ele de forma efetiva (RABELO et al., 2020).

Diante desse enfrentamento, relacionado as atividades remotas, faz ocorrer alto impacto para adaptações dessas mudanças (BRASIL, MEC, 2020), entre estas estão a falta de tempo, lazer e pouco convívio social, que conforme as experiências e no decorrer do curso surge a necessidade de reconhecimento como algo que deve ser superado, porém é importante se autopoliciar para que o cuidar do próximo não prevaleça sobre o cuidar de si (HIRSCH et al., 2018).

Dependendo da forma que as atividades são implementadas percebe-se insatisfações, provocadas pela percepção de baixo conhecimento teórico e prático adquirido por parte dos graduandos (HIRSCH et al., 2018). São considerados desencadeadores de estresse todo esse contexto apresentado, podendo levar a sintomas iniciais como o nervosismo, impaciência e irritabilidade (YOSETAKE et al., 2018).

Em estudantes de enfermagem, a prevalência dos sintomas de estresse identificado em estudo realizado na região Nordeste do Brasil, junto a 236 estudantes, correspondeu a um total de 73,3\% (COSTA et al., 2018). E segundo o teste de pontuação Perceived Stress Scale (PSS), o principal causador de estresse é a coerção da memória sobre a realização de tarefas diárias da faculdade, decorrentes na maioria das vezes da sistematização do curso acadêmico, acarretando em instabilidade emocional, física e social (YOSETAKE et al., 2018). 
Habitualmente, os estudantes de enfermagem já apresentam elevados graus de estresse, contudo, posteriormente a pandemia que provocou o isolamento social e consequentes mudanças nos hábitos de vida, impactando ainda mais na rotina acadêmica, tem-se como hipótese que essa condição de estresse está ainda mais acentuada, destarte, tem-se o seguinte questionamento: Estudantes de enfermagem estão apresentando elevados sintomas de estresse relacionado ao modo de ensino remoto durante a pandemia?

Trata-se de um estudo deveras relevante, uma vez que por ser uma condição nova, porém com tendência de permanência ainda por um longo período, é premente compreender os impactos do ensino remoto no contexto da formação do futuro profissional que estará atuando junto às pessoas em situação de adoecimento. Desse modo, essa pesquisa teve como objetivo estimar a prevalência de sintomas de estresse em estudantes universitários de enfermagem em uma Universidade de Maringá, Paraná (PR), durante as atividades remotas.

\section{METODOLOGIA}

Trata-se de um estudo quantitativo do tipo transversal e descritivo, realizado no período de novembro de 2020 à fevereiro de 2021. Decorrente da pandemia da COVID19 e por consequência do isolamento social, a coleta de dados foi realizada de forma remota, com participação de alunos da Universidade Cesumar, localizada em MaringáPR.

Participaram do estudo 75 estudantes do curso de graduação bacharel em enfermagem, independente do ano, sendo que nesta instituição a grade acadêmica corresponde a 4 anos. Como critério de inclusão foram selecionados apenas graduandos matriculados neste curso e Instituição, maiores de 18 anos, que realizaram as atividades remotas e aceitaram participar do estudo proposto, conforme Termo de Consentimento Livre e Esclarecido (TCLE).

A coleta de dados foi realizada de forma online, com a adaptação dos questionários no Google Forms, sendo utilizado a rede social WhatsApp ou e-mail, conforme a disponibilidade do universitário. Para tanto, foi solicitada autorização da coordenação do curso, bem como contato telefônico e e-mail dos alunos matriculados e em atividade remota, de todos as séries. 
De posse destas informações, foi então enviado uma mensagem/convite a participar do estudo, em que continha o título, objetivos, modo de participação, desobrigação do colaborador de se identificar ou ainda de responder o instrumento completamente, sendo assegurado a desistência em qualquer momento, sem que isso lhe acarretasse danos. Quando do aceite em participar, o respondente recebeu o Termo de Consentimento Livre e Esclarecido, para que formalizasse seu aceite e então tivesse acesso ao instrumento de coleta de dados. Este por sua vez foi confeccionado na plataforma Google Forms (GOOGLE, 2020). Os formulários Google permitem que o usuário colete e organize gratuitamente informações com grande variabilidade de tipos de resposta (GOOGLE, 2020).

O formulário possuiu três seções: dados sociodemográficos e psicossociais, seguida do rendimento acadêmico e aplicação da Perceived Stress Scale (PSS) (LUFT et al., 2007).

A primeira parte correspondeu a caracterização dos participantes quanto ao sexo, idade, cor, estado civil, filhos, renda familiar, com quem reside; se faz acompanhamento psicológico, uso de medicamento contínuo, substâncias psicoativas, uso de cigarro; se realiza atividade física ( 3 vezes por semana), lazer semanal; proporção de horas de sono e se trabalha.

A segunda parte composta por perguntas a respeito da opinião dos graduandos sobre o rendimento acadêmico: 1. Avaliação da média de nota no curso após início das aulas remotas (Inferior à 6.0, de 6.0 à 7.9 e de 8.0 à 10.0); 2. Como você descreveria seu desempenho acadêmico no momento? (Ruim, regular, bom, muito bom e ótimo). 3. Você considera que têm vivido alguma dessas situações estressoras relacionadas ao curso de enfermagem? (Múltipla escolha: carga horária, disciplinas complexas, estágios, excesso de atividades, falta de empatia por parte dos professores e outras). 4. Como você costuma lidar com essas situações estressoras abordadas anteriormente? (Bem; muito bem, nem bem e nem mal, mal e muito mal). 5. Com que frequência você encontra dificuldades no manejo do estresse? (Sempre, quase sempre, raramente, quase nunca e nunca) 6. Caso sinta estresse, quais as reações apresentadas? (Múltipla escolha: ansiedade, cansaço excessivo, depressão, dor, insônia, irritabilidade e outros).

A última foi a aplicação da PSS em versão brasileira, visto que essa possibilita mensurar o estresse percebido, composta por 14 perguntas, contendo cinco opções de respostas, sendo essas 0 (nunca), 1 (quase nunca), 2 (às vezes), 3 (quase sempre) e 4 
(sempre). Para análise das questões com relevância negativa aponta-se somatória normal e relevância positiva aponta-se somatória inversa $(0=4,1=3,2=2,3=1,4=0)$, podendo a resposta final de cada indivíduo equivaler de 0 à 56 (LUFT et al., 2007). Para melhor conclusão dos dados optou-se por subdividir os resultados finais em tercil, sendo esses, baixo (0-18), médio (19-37) e alto (38-56), e assim, observar qual a maior prevalência das respostas obtidas.

As respostas, por sua vez, foram armazenadas em planilhas (Google Sheets), permitindo serem visualizadas em formato de tabelas (GOOGLE, 2020). Após a coleta, os mesmos foram transcritos e contabilizados no software Microsoft Office Excel ${ }^{\circledR} 2010$. Para realização da análise da coleta utilizou-se frequências simples e porcentagens em tabelas, subdividindo-as em dados sociodemográficos e psicossociais, rendimento acadêmico, aplicação da PSS e escala de tercil, evidenciando os fatores e perfil que mais dominaram. Posteriormente os resultados foram discutidos à luz da literatura atual e pertinente.

O presente estudo foi aprovado pelo Comitê de Ética em Pesquisa com Seres Humanos, da Universidade Cesumar de Maringá-PR, sob $\mathrm{n}^{\circ}$ 4.194.905, CAAE: 35917220.6.0000.5539, agosto, 2020, respeitando a lei dos aspectos éticos da resolução do Conselho Nacional de Saúde (CNS) n. 466/2012.

\section{RESULTADOS}

Dos 75 participantes da pesquisa, no tocante das variáveis sociodemográficas e psicossociais, a mais relevante foi o público feminino (92\%). Em relação a faixa etária, a mais frequente foi de 16 a 25 anos (72\%). Quanto a etnia e estado civil, obtiveram maior prevalência a branca $(62,7 \%)$ e solteiro $(73,3 \%)$, respectivamente (Tabela 1$)$.

No que diz respeito a variável sobre possuir filhos, a maioria $(77,3 \%)$ dos acadêmicos não possuem, assim como não trabalham (60\%), possuem uma renda familiar superior a três salários mínimos $(49,3 \%)$, residem com a família $(93,3 \%)$, não realizam acompanhamento psicológico (81,3\%), não usam medicamento contínuo (56\%) e se automedicam, principalmente com analgésicos $(20,9 \%)$ (Tabela 1).

Ademais, em relação ao uso de substâncias psíquicas, as drogas lícitas $(48,8 \%)$ obtiveram maior predominância. A maioria dos estudantes pesquisados, não praticam 
atividade física semanal (64\%), porém grande parte realiza algum tipo de lazer (92\%), tendo principalmente de seis a oito horas diária de sono $(70,7 \%)$ (Tabela 1).

Tabela 1: Distribuição de variáveis sociodemográficas e psicossociais dos participantes. Maringá, PR, Brasil, 2021.

\begin{tabular}{|c|c|c|}
\hline Variáveis & $\mathbf{N}$ & $\%$ \\
\hline \multicolumn{3}{|l|}{ Sexo } \\
\hline Feminino & 69 & $92 \%$ \\
\hline Masculino & 6 & $8 \%$ \\
\hline \multicolumn{3}{|l|}{ Idade (anos) } \\
\hline $16-20$ & 27 & $36 \%$ \\
\hline $21-25$ & 27 & $36 \%$ \\
\hline $26-30$ & 5 & $6,7 \%$ \\
\hline $31-40$ & 14 & $18,7 \%$ \\
\hline$>41$ & 2 & $2,7 \%$ \\
\hline \multicolumn{3}{|l|}{ Etnia } \\
\hline Branco & 47 & $62,7 \%$ \\
\hline Pardo & 21 & $28 \%$ \\
\hline Negro & 5 & $6,7 \%$ \\
\hline Amarelo & 2 & $2,7 \%$ \\
\hline Indígena & 0 & $0 \%$ \\
\hline \multicolumn{3}{|l|}{ Estado civil } \\
\hline Solteiro & 55 & $73,3 \%$ \\
\hline Casado & 12 & $16 \%$ \\
\hline União estável & 8 & $10,7 \%$ \\
\hline Divorciado & 0 & $0 \%$ \\
\hline Viúvo & 0 & $0 \%$ \\
\hline \multicolumn{3}{|l|}{ Possui filhos? } \\
\hline Sim & 17 & $22,7 \%$ \\
\hline Não & & $77,3 \%$ \\
\hline \multicolumn{3}{|l|}{ Possui trabalho? } \\
\hline Sim & 30 & $40 \%$ \\
\hline Não & 45 & $60 \%$ \\
\hline \multicolumn{3}{|l|}{ Renda familiar } \\
\hline $\begin{array}{l}\text { Até } 1 \text { salário mínimo e } \\
\text { meio }\end{array}$ & 16 & $21,3 \%$ \\
\hline Até 3 salários mínimos & 37 & $49,3 \%$ \\
\hline $\begin{array}{l}\text { Superior a } 4 \text { salários } \\
\text { mínimos }\end{array}$ & 22 & $29,3 \%$ \\
\hline \multicolumn{3}{|l|}{ Com quem reside? } \\
\hline Sozinho & 3 & $4 \%$ \\
\hline Família & 70 & $93,3 \%$ \\
\hline Amigos & 2 & $2,7 \%$ \\
\hline \multicolumn{3}{|c|}{ Faz acompanhamento psicológico? } \\
\hline Sim & 14 & $18,7 \%$ \\
\hline Não & 61 & $81,3 \%$ \\
\hline \multicolumn{3}{|c|}{ Faz uso de medicação contínua? } \\
\hline Sim & 33 & $44 \%$ \\
\hline
\end{tabular}


Se automedica com quais medicamentos? *

$\begin{array}{lll}\text { Analgésico/antitérmico } & 41 & 20,9 \% \\ \text { Descongestionante nasal } & 21 & 10,7 \% \\ \text { Relaxante muscular } & 34 & 17,3 \% \\ \text { Anticoncepcional } & 22 & 11,2 \% \\ \text { Antiinflamatório } & 27 & 13,8 \% \\ \text { Antialérgico } & 33 & 16,8 \% \\ \text { Outros } & 18 & 9,2 \%\end{array}$

Utiliza substâncias psíquicas? *

$\begin{array}{lll}\text { Drogas lícitas } & 20 & 48,8 \% \\ \text { Drogas ilícitas } & 6 & 14,6 \% \\ \text { Psicotrópicos } & 14 & 34,1 \% \\ \text { Outros } & 1 & 2,4 \%\end{array}$

\section{Atividade física}

Sim 27

Não 48

$27 \quad 36 \%$

Faz uso de tabaco?

Sim 5

Não 70

$48 \quad 64 \%$

Realiza lazer semanal?

Sim

69

$6,7 \%$

Não

Horas de sono diária

Menos de 6 horas

$14 \quad 18,7 \%$

6-8 horas

$92 \%$

$8 \%$

Acima de 8 horas $\quad 8 \quad 10,7 \%$

\begin{tabular}{cc}
\hline Total & 75 \\
\hline & *Perguntas que podiam marcar mais de uma alternativa como resposta. \\
& Fonte: Dados da pesquisa
\end{tabular}

Verificou-se que diante das atividades remotas o rendimento acadêmico predominou com média de nota de 8.0 à 10.0 (53,3\%) e o desempenho em sua maioria foi considerado bom (32\%). Outrossim, enfatizando vivências estressoras, consideram como causas o excesso de atividades $(31,7 \%)$ e disciplinas complexas $(25,2 \%)$, tratando nem bem nem mal estes fatos $(39,1 \%)$, porém apresentam dificuldade quase sempre no manuseio do estresse (50,7\%), relacionada principalmente por ansiedade (23,6\%), irritabilidade $(22,8 \%)$ e cansaço excessivo $(19,1 \%)$ (Tabela 2$)$.

A Perceived Stress Scale (PSS), tem como resultados pontuações variáveis de 0 a 56 pontos (LUFT et. al., 2007). Na aplicação da PSS neste estudo, avaliando-se as somatórias das respostas obtidas, resultou-se como mínima o valor correspondente de 13 pontos e máxima 56. 
Tabela 2: Distribuição do rendimento autorreferido do acadêmico de enfermagem durante as atividades remotas. Maringá, PR, Brasil, 2021.

\begin{tabular}{|c|c|c|}
\hline Rendimento acadêmico & $\mathbf{N}$ & $\%$ \\
\hline \multicolumn{3}{|c|}{ Média de nota no curso após início das aulas remotas? } \\
\hline Inferior à 6.0 & 3 & $4 \%$ \\
\hline De 6.0 à 7.9 & 32 & $42,7 \%$ \\
\hline De 8.0 à 10.0 & 40 & $53,3 \%$ \\
\hline \multicolumn{3}{|c|}{ Como você descreveria seu desempenho acadêmico no momento? } \\
\hline Ruim & 14 & $18,7 \%$ \\
\hline Regular & 23 & $30,7 \%$ \\
\hline Bom & 24 & $32 \%$ \\
\hline Muito bom & 13 & $17,3 \%$ \\
\hline Ótimo & 1 & $1,3 \%$ \\
\hline \multicolumn{3}{|c|}{$\begin{array}{l}\text { Você considera que tem vivido alguma dessas situações estressoras relacionadas } \\
\text { ao curso de enfermagem? } *\end{array}$} \\
\hline Carga horária & 18 & $14,6 \%$ \\
\hline Disciplinas complexas & 31 & $25,2 \%$ \\
\hline Estágios & 14 & $11,4 \%$ \\
\hline Excesso de atividades & 39 & $31,7 \%$ \\
\hline $\begin{array}{l}\text { Falta de empatia por parte dos } \\
\text { professores }\end{array}$ & 13 & $10,6 \%$ \\
\hline Outras & 8 & $6,5 \%$ \\
\hline
\end{tabular}

Como você costuma lidar com as situações estressoras abordadas anteriormente?

$\begin{array}{lll}\text { Bem } & 19 & 27,5 \% \\ \text { Muito bem } & 3 & 4,3 \% \\ \text { Nem bem, nem mal } & 27 & 39,1 \% \\ \text { Mal } & 18 & 26,1 \% \\ \text { Muito mal } & 2 & 2,9 \%\end{array}$

Com que frequência você encontra dificuldades no manejo do estresse?

$\begin{array}{lll}\text { Sempre } & 16 & 21,3 \% \\ \text { Quase sempre } & 38 & 50,7 \% \\ \text { Raramente } & 19 & 25,3 \% \\ \text { Quase nunca } & 2 & 2,7 \% \\ \text { Nunca } & 0 & 0 \%\end{array}$

Caso tenha estresse, quais as reações apresentadas? *

\begin{tabular}{lll} 
Ansiedade & 58 & $23,6 \%$ \\
Cansaço excessivo & 47 & $19,1 \%$ \\
Depressão & 16 & $6,5 \%$ \\
Dor & 23 & $9,3 \%$ \\
Insônia & 37 & $15 \%$ \\
Irritabilidade & 56 & $22,8 \%$ \\
Outras & 9 & $3,7 \%$ \\
\hline Total & 75 & $100 \%$ \\
\hline \multicolumn{3}{c}{ *Perguntas que podiam marcar mais de uma alternativa como resposta. } \\
& Fonte: Dados da pesquisa
\end{tabular}

Correspondente ao tercil, verificou-se que o nível de estresse mais acentuado foi o médio (72\%), posteriormente o elevado $(25,3 \%)$ e baixo $(2,7 \%)$ (Tabela 3$)$ 
Tabela 3: Níveis de estresse de acordo com a pontuação das respostas entre acadêmicos de enfermagem. Maringá, PR, Brasil, 2021.

\begin{tabular}{llll}
\hline $\begin{array}{l}\text { Nível de } \\
\text { estresse }\end{array}$ & Tercil & Número de participantes & $\%$ \\
\hline Baixo & $0-18$ pontos & 2 & $2,7 \%$ \\
Médio & $19-37$ pontos & 54 & $72 \%$ \\
Elevado & $38-56$ pontos & 19 & $25,3 \%$ \\
\hline Total & 56 & 75 & 100 \\
\hline
\end{tabular}

Fonte: Dados da pesquisa

Entre as 14 perguntas, as com conotação positiva (4, 5, 6, 7, 9, 10 e 13), sendo estas somadas de maneira invertida, $0=4,1=3,2=2,3=1$ e $4=0$, em todas, a mediana apresentou-se como 2. Em contrapartida, as de conotação negativa (1, 2, 3, 8, 11, 12 e 14), as quais são somadas diretamente, a mediana que predominou foi a 3 nas perguntas 1, 2, 3 e 11, posteriormente a mediana 2 nas questões 8 e 14, e por fim, na questão 12 obteve mediana máxima, igual a 4 (Tabela 4).

Tabela 4: Dados da PSS em acadêmicos de enfermagem, em ordem crescente de pergunta. Maringá, PR, Brasil, 2021.

\begin{tabular}{|c|c|c|c|c|c|}
\hline $\begin{array}{l}\text { Item } \\
\mathbf{N}^{\circ}\end{array}$ & $\begin{array}{l}\text { No último mês com que } \\
\text { frequência você tem... }\end{array}$ & Mín/Máx & Média & Mediana & $\begin{array}{l}\text { Desvio- } \\
\text { padrão }\end{array}$ \\
\hline 1 & $\begin{array}{l}\text { ficado triste por causa de algo que } \\
\text { aconteceu inesperadamente? }\end{array}$ & $1 / 2$ & 1,5 & 3 & 0,7 \\
\hline 2 & $\begin{array}{l}\text { se sentido incapaz de controlar as } \\
\text { coisas importantes em sua vida? }\end{array}$ & $0 / 2$ & 1 & 3 & 1,4 \\
\hline 3 & se sentido nervoso e "estressado"? & $1 / 3$ & 2 & 3 & 1,4 \\
\hline 4 & $\begin{array}{l}\text { tratado com sucesso os problemas } \\
\text { difíceis da vida? }\end{array}$ & $0 / 2$ & 1 & 2 & 1,4 \\
\hline 5 & $\begin{array}{l}\text { sentido que está lidando bem com } \\
\text { as mudanças importantes que estão } \\
\text { ocorrendo em sua vida? }\end{array}$ & $0 / 3$ & 1,5 & 2 & 2,1 \\
\hline 6 & $\begin{array}{l}\text { se sentido confiante na sua } \\
\text { habilidade de resolver problemas } \\
\text { pessoais? }\end{array}$ & $0 / 3$ & 1,5 & 2 & 2,1 \\
\hline 7 & $\begin{array}{l}\text { sentido que as coisas estão } \\
\text { acontecendo de acordo com a sua } \\
\text { vontade? }\end{array}$ & $0 / 2$ & 1 & 2 & 1,4 \\
\hline
\end{tabular}




\begin{tabular}{|c|c|c|c|c|c|}
\hline $\begin{array}{l}\text { Item } \\
\mathbf{N}^{\circ}\end{array}$ & $\begin{array}{l}\text { No último mês com que } \\
\text { frequência você tem... }\end{array}$ & Mín/Máx & Média & Mediana & $\begin{array}{l}\text { Desvio- } \\
\text { padrão }\end{array}$ \\
\hline 8 & $\begin{array}{l}\text { achado que não conseguiria lidar } \\
\text { com todas as coisas que você tem } \\
\text { que fazer? }\end{array}$ & $0 / 2$ & 1 & 2 & 1,4 \\
\hline 9 & $\begin{array}{l}\text { conseguido controlar as irritações } \\
\text { em sua vida? }\end{array}$ & $0 / 2$ & 1 & 2 & 1,4 \\
\hline 10 & $\begin{array}{l}\text { sentido que as coisas estão sob o } \\
\text { seu controle? }\end{array}$ & $0 / 2$ & 1 & 2 & 1,4 \\
\hline 11 & $\begin{array}{l}\text { ficado irritado porque as coisas } \\
\text { que acontecem estão fora do seu } \\
\text { controle? }\end{array}$ & $0 / 3$ & 1,5 & 3 & 2,1 \\
\hline 12 & $\begin{array}{l}\text { se encontrado pensando sobre as } \\
\text { coisas que tem que fazer? }\end{array}$ & $1 / 4$ & 2,5 & 4 & 2,1 \\
\hline 13 & $\begin{array}{l}\text { conseguido controlar a maneira } \\
\text { como gasta seu tempo? }\end{array}$ & $0 / 2$ & 1 & 2 & 1,4 \\
\hline 14 & $\begin{array}{l}\text { sentido que as dificuldades se } \\
\text { acumulam a ponto de você } \\
\text { acreditar que não pode superá- } \\
\text { las? }\end{array}$ & $0 / 2$ & 1 & 2 & 1,4 \\
\hline \multicolumn{2}{|c|}{ PSS* total: Somatória de pontos } & $0 / 4$ & 18,5 & 34 & 21,7 \\
\hline
\end{tabular}

\section{Fonte: Dados da pesquisa}

O total da mediana correspondeu a 34 , sendo que as perguntas negativas se sobressaíram às positivas, evidenciando a presença do nível médio de estresse percebido na aplicação da PSS nos graduandos de enfermagem, em meio as aulas remotas, consequentes do isolamento social que foi provocado pela COVID-19 (Tabela 4).

Analisando-se os comparativos entre os dados sociodemográficos e resultados da PSS, houve diferenças na média de pontuação PSS em termos de trabalho, sendo observado grande discrepância. Os colaboradores do estudo que relataram trabalhar e estudar, obtiveram maior resultado $(48,4)$ quando comparados aos que não trabalham $(22,9)$. Em relação as demais correlações dos dados, não foram consideradas diferenças, pois os resultados não foram suficientes para serem comparados (Tabela 4). 


\section{DISCUSSÃO}

Neste estudo, graduandos de enfermagem foram avaliados no tangente de níveis de estresse durante a pandemia, devido mudança no estilo do ensino presencial para remoto, por meio de questionário eletrônico com a aplicação da Perceived Stress Scale (PSS). Com base nos dados levantados, evidenciou-se sintomas de nível médio de estresse, o que corrobora com outros estudos realizados com estudantes de enfermagem, avaliando o impacto da COVID-19 na saúde mental (ASLAN; PEKINCE, 2020; SUBBA et al., 2020; ENSALEM et al., 2020).

O toque de recolher gerado pela pandemia, provocou a diminuição de atividades sociais, exercícios físicos, além de irregularidades alimentares e no sono, sendo esses possíveis fenômenos que também levaram ao aumento do estresse nos estudantes, porém mesmo apresentando tédio por ficarem restritos em casa, a sensação de segurança foi maior (ASLAN; PEKINCE, 2020).

Analisando-se questões relacionadas ao sexo, o feminino foi predominante entre os respondentes, fato este relacionado à prevalência de mulheres cursando Enfermagem. É notório que as mulheres apresentam sinais de fragilidade emocional, como maior incerteza, pior autoconceito e sintomas de estresse quando comparado ao masculino, especialmente frente às situações estressoras, tais como tem sido o ensino remoto (GOMÉS et al., 2020; ASLAN; PEKINSE, 2020; SHAH et al., 2020; UĞURLU et al., 2020).

A faixa etária dos graduandos prevaleceu entre 16 a 25 anos, onde também foi verificado um elevado relato de ansiedade, em concordância com estudos similares (ASLAN; PEKINCE, 2020; SUBBA et al., 2020; GOMÉS et al.,2020), compreendendose que esse público possui menor habilidade de enfrentamento psicológico, logo, outro estudo mostra a redução da pontuação da PSS conforme a idade dos acadêmicos aumenta (MUVID et al., 2021).

$\mathrm{Na}$ presente pesquisa, cerca de $40 \%$ dos participantes trabalham, tendo como média na PSS a pontuação de 48,4, correspondendo ao tercil elevado de estresse, assim como outra pesquisa apontou que os alunos que possuem emprego remunerado optaram por este devido ao fato de passarem por restrições financeiras. Porém, a pesquisa relata como impacto negativo a redução do tempo para se dedicar aos estudos, baixa produtividade, sobrecarga e estresse. Por outro lado, o fato de trabalharem, fez com que 
os estudantes aprendessem a controlar melhor o tempo, passassem a se esforçar mais, e consequentemente controlar a sensação de estresse em meio ao trabalho, aumentando assim, suas habilidades gerenciais do dia a dia (MITCHELL, 2020; WARNER et al., 2020).

Observa-se que predominou o uso de analgésicos em relação a automedicação, podendo ser causada pela presença de dores de cabeça, cólicas menstruais e febre, associada a falta de tempo para procurar serviços médicos, ocasionada pela sobrecarga das atividades acadêmicas. Outro fator que corrobora para se automedicar corresponde ao fato de os graduandos já terem passado pelos mesmos sintomas anteriormente e com a automedicação obtiveram alívio, junto a isso envolvem seus conhecimentos sobre os sintomas e tratamento correspondente (ELSALEM et al., 2020; FAQIHI; SAYED, 2021).

Em relação ao sono, constatou-se na literatura, que em meio a pandemia houve redução da quantidade das horas de sono (ELSALEM et al., 2020; ASLAN; PEKINCE, 2020). Em contrapartida, na presente pesquisa, mesmo em contexto de pandemia e isolamento social, os estudantes de enfermagem dormem de 6 a 8 horas diárias, ou seja, mantiveram boa qualidade de sono.

Atrelado ao conceito de saúde mental, evidenciou-se sintomas leves de ansiedade (UĞURLU et al., 2020), assim como alunos apontaram essa, uma das mais frequentes reações apresentadas pelo estresse. Ademais, estudos apontam que acadêmicos em situação de vulnerabilidade social e econômica, tais como dificuldades financeiras, familiares ou emocionais evidencia-se estresse mais elevado. Entre os colaboradores do presente estudo tais informações não foram levantadas, contudo, é possível que situações como estas também sejam vivenciadas, potencializando os sintomas de ansiedade referidos (GOMÉS et al.,2020).

O excesso de atividade, disciplinas complexas e carga horária extensa são consideradas pelos estudantes como as principais situações estressoras, confirmado por outro estudo, em que as mesmas corroboram para sintomas de esgotamento físico e mental, como: dores de cabeça e musculares, estresse e insônia (GALDINO et al., 2020).

Estudo com 155 estudantes universitários do curso de graduação em enfermagem, do estado do Amazonas/Brasil, verificou-se a pontuação de média intensidade de estresse na Escala de Avaliação de Estresse em Estudantes de Enfermagem (AEEE), predominando os tangentes de realização das atividades práticas, ambiente acadêmico e 
gerenciamento de tempo. Outrossim, a pontuação de alta intensidade de estresse prevaleceu a comunicação e formação profissional (FONSECA et al., 2019).

No que se refere, aos diferentes anos curriculares, os níveis de estresse dos alunos do primeiro e quarto anos são maiores, sendo isso justificado pelo fato do primeiro ano estar iniciando as atividades acadêmicas e possuir menor conhecimento e habilidades

profissionais e o quarto ano apresentar-se ansioso, devido a conclusão do curso e a proximidade com a responsabilidade profissional (ASLAN; PEKINCE, 2020; FONSECA et al., 2019).

Em estudo realizado com 207 estudantes de enfermagem, através de questionário online, outras intervenções foram identificadas em decorrência do bloqueio, provocado pela pandemia, o qual afetou a produtividade da educação, em que 99,5\% dos alunos relataram atraso acadêmico e 57\% consideraram o ensino ineficiente, corroborando para a confirmação da pesquisa em proposta (SUBBA et al., 2020).

Portanto, é possível inferir com base nos resultados levantados, que a prevalência dos sintomas de estresse ocorre em graduandos de enfermagem em nível moderado, além da presença de sintomas de ansiedade, sendo que a maior parte dos pesquisados eram do sexo feminino. Ademais, alunos que trabalham apresentaram nível mais elevado desse distúrbio. Quanto aos alunos que possuem o hábito de se automedicar, observa-se como uso principal os analgésicos, para alívio de sintomas que condizem com os do estresse. Ainda, o excesso de atividades e complexidade das disciplinas são situações vivenciadas que provocam maior impacto na prevalência dos sintomas de estresse.

\section{CONCLUSÃO}

Com base nos resultados deste estudo, a maioria dos estudantes de enfermagem apresentaram nível moderado de estresse percebido em meio ao período atual de confinamento, sendo esse refletido por comportamentos ansiosos, irritabilidade e cansaço excessivo, gerados principalmente pelo excesso de atividades, demandadas pelo curso de graduação em enfermagem.

O manejo do estresse foi considerado quase sempre difícil de ser controlado, porém independente disso, as médias das notas mantiveram-se elevadas, demonstrando assim, que mesmo diante da pandemia houve grande empenho por parte dos alunos, 
podendo esse estar atrelado ao fato dos universitários estarem restritos em suas casas e limitarem-se de atividades sociais.

Com toda mudança atual e notícias da mídia em relação a gravidade do momento e sobrecarga da profissão de enfermagem, a consciência dos estudantes pode estar sendo sensibilizada, levando estes a possíveis sensações desanimadoras sobre a profissão. Conclui-se assim, a necessidade de maior atenção e planejamento sobre a saúde mental desses graduandos, além de promover o esclarecimento de dúvidas e desentendimentos informativos.

Entre os empasses destacados, deve-se atentar à percepção do aluno a respeito da pandemia atual, uma vez que pode prejudicar seu convívio social e futura profissão, ainda mais por se tratar de uma profissão em que sua maioria necessita da qualidade do trabalho em equipe e equilíbrio emocional, em que o objetivo principal é prestar assistência.

De acordo com a pesquisa realizada, foi analisada a escassez de estudos diante da temática, enfatizando a importância em desenvolver novos estudos que mostrem os fatores corroborativos para desequilíbrios mentais em estudantes universitários de enfermagem no contexto de pandemia e atividades acadêmicas remotas, a fím de proporcionar uma atenção mais assertiva voltada a saúde mental destes, evitando-se assim, distúrbios psicológicos.

\section{REFERÊNCIAS}

AHMED, M. Z. et al. Epidemic of COVID-19 in China and associated Psychological Problems. Asian Journal of Psychiatry, v. 51, 2020. Disponível em: https://www.sciencedirect.com/science/article/abs/pii/S1876201820302033?via\%3Dihu b. Acesso em: 12 jun. 2021.

ASLAN, H.; PEKINCE, H. Nursing students' views on the COVID-19 pandemic and their

percieved stress levels. Perspectives in Psychiatric Care, v.57, ed.2: 695-701, 2020. Disponível em: https://onlinelibrary.wiley.com/doi/full/10.1111/ppc.12597. Acesso em: 29 maio 2021.

BEZERRA, A. C. V. et al. Fatores Associados ao Comportamento da População durante o Isolamento Social na Pandemia de COVID-19. Ciência e Saúde Coletiva, v. 25, n. 1: 2411-2421, 2020. Disponível em: https://www.scielo.br/j/csc/a/9g4hLHkSSW35gYsSpggz6rn/?lang=pt. Acesso em: 10 maio 2020. 
BRASIL. LIMA, L. MEC autoriza ensino a distância em cursos presenciais. Ministério da Educação, 2020. Disponível em:

http://portal.mec.gov.br/component/content/article?id=86441. Acesso em: 10 maio 2020.

BRASIL. Ministério da Saúde. Coronavírus (COVID-19), 2020. Disponível em: https://coronavirus.saude.gov.br/. Acesso em: 12 maio 2020.

BRASIL. Portaria n ${ }^{\circ}$ 343, de 17 de março de 2020. Ministério da educação. Diário Oficial da União, 2020. Disponível em: http://www.in.gov.br/en/web/dou/-/portaria-n343-de-17-de-marco-de-2020-248564376. Acesso em: 10 maio 2020.

CASTELLA, M. et al. Features, Evaluation and Treatment Coronavirus (COVID-19). StatPearls, 2020. Disponível em:

https://pubmed.ncbi.nlm.nih.gov/32150360/?from_term=Features\%2C+Evaluation+and +Treatment+Coronavirus+\%28COVID-19\%29\&from_pos=1. Acesso em: 11 maio 2020 .

COSTA, C. R. B. et al. Estresse entre estudantes de graduação em enfermagem: Associação de características sociodemográficas de acadêmicas. Saúde e Pesquisa, v. 11, n. 3: 475-482, 2018. Disponível em:

https://periodicos.unicesumar.edu.br/index.php/saudpesq/article/view/6765/3282.

Acesso em: 11 maio 2020.

ELSALEM, L. Stress and behavioral changes with remote E-exams during the Covid-19 pandemic: A cross-sectional study among undergraduates of medical sciences. Annals of Medicine and Surgery, v. 60: 271-279, 2020. Disponível em:

https://www.sciencedirect.com/science/article/pii/S2049080120304131?via\%3Dihub. Acesso em: 29 maio 2021.

FAQIHI, A. M. A.; SAYED, S. F. Self-medication practice with analgesics (NSAIDs and acetaminophen), and antibiotics among nursing undergraduates in University College Farasan Campus, Jazan University, KSA. Annales Pharmaceutiques Françaises, v. 79, ed. 3: 275-285, 2021. Disponível em: https://www.sciencedirect.com/science/article/abs/pii/S0003450920301280?via\%3Dihu b. Acesso em: 10 jul. 2021.

FONSECA, J. R. F. et al. Associação dos fatores de estresse e sintomas depressivos com o desempenho acadêmico de estudantes de enfermagem. Revista da Escola de Enfermagem da USP, 2019. Disponível em: https://www.scielo.br/j/reeusp/a/VvXkZqWYVfBxqGMwSGxpP8S/?lang=pt. Acesso em: 10 jul. 2021.

GALDINO, M. J. Q. et al. Burnout among nursing students: a mixed method study. Investigación y Educación en Enfermería, v. 38, n. 1, 2020. Disponível em: https://revistas.udea.edu.co/index.php/iee/article/view/341298. Acesso em: 10 jul. 2021.

GALLEGO-GÓMEZ, J. I. et al. The COVID-19 Pandemic and Its Impact on Homebound Nursing Students. International Journal of Environmental Research 
and Public Health, v. 17, n. 20, 2020. Disponível em: https://www.mdpi.com/16604601/17/20/7383. Acesso em: 29 maio 2021.

GOOGLE. Clear Google Drive space \& amp; increase storage. 2017. Disponível em: https://www.google.com.br/drive/apps.html. Acesso em: 08 maio 2020.

GROVER, S. et al. Psychological impact of COVID-19 lockdown: An online survey from India. Indian Journal of Psychiatry, v. 62, n. 4: 354-362, 2020. Disponível em: https://www.ncbi.nlm.nih.gov/pmc/articles/PMC7597717/. Acesso em: 12 jun. 2021.

HIRSCH, C. D. et al. Fatores percebidos pelos acadêmicos de enfermagem como desencadeadores do estresse no ambiente formativo. Texto e Contexto Enfermagem, v. 27, n. 1, 2018. Disponível em:

https://pdfs.semanticscholar.org/34b2/8813b856b08c0fee9991f72255d7535c9411.pdf. Acesso em: 11 maio 2020.

LIPP, M. E. N. Manual Do Inventário de Sintomas de Stress para Adultos de Lipp (ISSL). São Paulo: Casa do Psicólogo; 2000.

LIPP, M. E. N. Pesquisas Sobre Stress no Brasil. Campinas, SP: Papirus, 1997.

LOPES, L. S. et al. Estudo sobre a qualidade de vida dos estudantes da universidade federal de Rondonópolis, MT utilizando dados comportamentais. Biodiversidade, v. 2, n. 18, 2019. Disponível em:

http://www.periodicoscientificos.ufmt.br/ojs/index.php/biodiversidade/article/view/869 4/5912. Acesso em: 11 maio 2020.

LUFT, C. D. B. et al. Versão brasileira da Escala de Estresse Percebido: tradução e validação para idosos. Revista de Saúde Pública, v. 41, n. 4: 606-615, 2007.

Disponível em:

https://www.scielo.br/j/rsp/a/bgpXDHZXQXNqVS8JLnLdLhr/?lang=pt. Acesso em: 11 maio 2020.

MITCHELL, J. Juggling employment and studies: Nursing students' perceptions of the influence of paid employment on their success. Nurse Education Today, v. 92, 2020. Disponível em:

https://www.sciencedirect.com/science/article/abs/pii/S0260691719307348?via\%3Dihu b. Acesso em: 10 jul. 2021.

MUVID, Y. et al. Estrés percibido en estudiantes de enfermería durante el confinamiento obligatorio por Covid-19. Revista Cuidarte, v. 12, n. 2, 2021. Disponível em: https://revistas.udes.edu.co/cuidarte/article/view/1330. Acesso em: 29 maio 2021.

OPAS: Folha Informativa-COVID-19 (doença causada pelo novo coronavírus). Organização Pan-Americana da Saúde, 2020. Disponível em: https://www.paho.org/bra/index.php?option=com_content $\&$ view $=$ article $\& i d=6101:$ covi d19\&Itemid=875\#historico. Acesso em: 10 maio 2020. 
RABELO, L. M. et al. Estresse e a sua influência em acadêmicos de enfermagem: uma revisão. Revista Liberum Accessum, v. 1, n. 1, 2020. Disponível em: http://revista.liberumaccesum.com.br/index.php/RLA/article/view/20. Acesso em: 11 maio 2020.

SHAH, S. M. A. et al. Prevalence, Psychological Responses and Associated Correlates of Depression, Anxiety and Stress in a Global Population, During the Coronavirus Disease (COVID-19) Pandemic. Community Mental Health Journal, v. 57: 101-110, 2021. Disponível em: https://link.springer.com/article/10.1007/s10597-020-00728-y. Acesso em: 29 maio 2021.

SUBBA, R. et al. Psychological Impact and Coping Strategies of Nursing Students during the Outbreak of Coronavirus Disease in Nepal. Asian Journal of Multidisciplinary Studies, v. 8, n. 9, 2020. Disponível em: https://www.researchgate.net/publication/344201230_Psychological_Impact_and_Copi ng_Strategies_of_Nursing_Students_during_the_Outbreak_of_Coronavirus_Disease_in _Nepal. Acesso em: 29 maio 2021.

UĞURLU, Y. K. et al. The examination of the relationship between nursing students' depression, anxiety and stress levels and restrictive, emotional, and external eating behaviors in COVID-19 social isolation process. Perspectives in Psychiatric Care, v. 57: 507-516, 2020. Disponível em:

https://onlinelibrary.wiley.com/doi/10.1111/ppc.12703. Acesso em: 29 maio 2021.

WARNER, A. et al. The relationship among BSN students' employment, educational, and health-related characteristics and semester grades: A multi-site study. Journal of Professional Nursing, v. 36, ed. 5: 308-316, 2020. Disponível em: https://www.sciencedirect.com/science/article/abs/pii/S8755722320300053?via \%3Dihub. Acesso em: 10 jul. 2021.

YOSETAKE, A. L. et al. Estresse percebido em graduandos de enfermagem. Revista Eletrônica Saúde Mental Álcool e Drogas, v. 14, n. 2: 117-124, 2018. Disponível em: http://www.periodicos.usp.br/smad/article/view/155638/151281. Acesso em: 11 maio 2020. 